\title{
Diagnostic performance of two molecular assays for the detection of vaginitis in symptomatic women
}

\author{
Alexandra Thompson $^{1} \cdot$ Karen Timm $^{2} \cdot$ Noelle Borders $^{3} \cdot$ Liz Montoya $^{3} \cdot$ Karissa Culbreath $^{1,2}$ (D)
}

Received: 30 May 2019 / Accepted: 26 August 2019 / Published online: 9 September 2019

(C) The Author(s) 2019

\begin{abstract}
The three main causes of vaginitis are bacterial vaginosis (BV), vulvovaginal candidiasis (VVC), and trichomoniasis (TV). Two multiplex assays are commercially available for detection of DNA from organisms associated with vaginitis: BD Affirm ${ }^{\mathrm{TM}}$ VPIII Microbial Identification Test (Affirm) and BD MAX ${ }^{\mathrm{TM}}$ Vaginal Panel (MAX VP). Here, the performance of MAX VP was compared to that of Affirm, which was considered the standard of care. Four vaginal swabs were collected from each subject with the following: BD Affirm ${ }^{\mathrm{TM}}$ VPIII Ambient Temperature Transport System (ATTS), BD MAX ${ }^{\mathrm{TM}}$ UVE Specimen Collection Kit, Hologic Aptima ${ }^{\circledR}$ Vaginal Swab Specimen Collection Kit, and BD ESwab ${ }^{\mathrm{TM}}$ collection and transport system (ESwab). Candida culture, Gram stain followed by Nugent scoring, and the Hologic Aptima® Trichomonas vaginalis assay were used for discordant analysis. Results were considered true positive if there were at least two tests positive for any vaginitis target. A total of 200 symptomatic women were evaluated in the study. The sensitivity and specificity of MAX VP for BV was $96.2 \%$ and $96.1 \%$, respectively, compared to $96.2 \%$ and $81.6 \%$ for Affirm. The sensitivity and specificity of MAX VP for Candida spp. was $98.4 \%$ and $95.4 \%$, respectively, compared to $69.4 \%$ and $100 \%$ for Affirm. MAX VP and Affirm showed $100 \%$ concordance for detection of TV. These results demonstrate improved accuracy of MAX VP compared to Affirm for the detection of BV and Candida spp. and no difference for detection of TV between the two tests.
\end{abstract}

Keywords Vaginitis · Trichomonas $\cdot$ Sexually transmitted infection · Molecular diagnostics

\section{Introduction}

Vaginitis encompasses a spectrum of conditions that cause vaginal and vulvar symptoms, including thickened or malodorous discharge, dysuria, itching, and general discomfort [1]. These complaints are the cause of ten million office visits per year [2]. Vulvovaginal candidiasis (VVC), trichomoniasis (TV), and bacterial vaginosis (BV) are three distinct infectious syndromes collectively classified as vaginitis causes [1]. VVC involves fungal infections of the vagina and is most commonly caused by Candida albicans; however, other species

Karissa Culbreath

Karissa.culbreath@tricore.org

1 University of New Mexico School of Medicine, Albuquerque, NM, USA

2 TriCore Reference Laboratories, Albuquerque, NM, USA

3 Department of Obstetrics and Gynecology, University of New Mexico School of Medicine, Albuquerque, NM, USA including $C$. tropicalis and $C$. glabrata are also implicated in VVC [3]. TV is a sexually transmitted infection caused by the flagellated protozoan Trichomonas vaginalis, which infects the vaginal epithelium [4]. The etiology of BV continues to evolve; however, symptomatic infection is generally associated with an imbalance in the vaginal flora resulting from a decrease in one or more species of the Lactobacillus genus with a concomitant increase of BV-associated bacteria including Gardnerella vaginalis, Atopobium vaginae, or other anaerobic bacteria $[5,6]$.

Accurate diagnosis and management of vaginitis is challenging due to the nonspecific nature of patient-reported symptoms, which may overlap with multiple etiologic agents that do not consistently predict the underlying cause(s) [7]. Inclinic diagnostic methods lack sensitivity and specificity for the detection and differentiation of causative agents of vaginitis [8]. Recurrent infections are common, requiring multiple clinic visits, which may be attributed to initial misdiagnosis of these syndromes [9]. Treatments for these conditions are different, which can contribute to repeat visit(s) if the initial diagnosis is incorrect [10]. Although morbidity may be low, 
there are significant risks associated with misdiagnosis of vaginitis. BV and TV have been linked to adverse pregnancy outcomes (preterm labor and premature rupture of membranes), pelvic inflammatory disease, and increased risk of transmission and acquisition of STIs, including HIV [11-14]. Therefore, careful history and examination, combined with accurate testing, is essential for a correct diagnosis and appropriate treatment course for patients [1].

The BD Affirm ${ }^{\mathrm{TM}}$ VPIII Microbial Identification Test (Affirm, Becton, Dickinson and Company, Sparks, MD) is a DNA probe hybridization test used to detect Gardnerella vaginalis, Candida spp. (including C. albicans, C. glabrata, C. kefyr, C. krusei, C. parapsilosis, and C. tropicalis), and T. vaginalis [15]. BD MAX ${ }^{\mathrm{TM}}$ Vaginal Panel (MAX VP; Becton, Dickinson and Company, Quebec, Canada) uses real-time PCR for the amplification of specific DNA targets to differentially detect (a) bacterial vaginosis through algorithmic analysis of lactobacilli (L. crispatus and L. jensenii) and bacteria involved in BV ( $G$. vaginalis, A. vaginae, Megasphaera-1, and BVAB-2); (b) Candida group (C. albicans, C. tropicalis, C. parapsilosis, and C. dubliniensis), C. glabrata, and C. krusei; and (c) Trichomonas vaginalis. Previous studies have described the performance of MAX VP compared to clinical diagnosis and nonamplification methods $[8,16]$. In this study, we performed a clinical evaluation of the performance of MAX VP compared to Affirm, the standard of care, for the detection of putative vaginal pathogens in symptomatic women.

\section{Methods}

\section{Study design}

A total of 215 symptomatic women presented to four obstetrics and gynecology clinics in the University of New Mexico Health Systems and consented to study participation. Patients were excluded from the study if they did not meet the inclusion criteria or did not consent to participate. Of the 215 women enrolled, 15 were excluded (10 duplicate patients; 1 incorrect specimen collection; 2 consent forms not completed; 2 unable to provide a specimen). The study was conducted under the approval of the Institution Review Board of the University of New Mexico.

\section{Sample collection and vaginitis assays and patient clinical data}

A total of four vaginal swab specimens per patient were collected for the study. These specimens included (1) BD Affirm $^{\mathrm{TM}}$ VPIII Ambient Temperature Transport System (Becton, Dickinson and Company, Sparks, MD), (2) BD MAX $^{\mathrm{TM}}$ UVE Specimen Collection Kit (Becton, Dickinson and Company, Sparks, MD), (3) Hologic Aptima ${ }^{\circledR}$ Vaginal Swab Collection Kit (Hologic Inc., San Diego, CA), and (4) BD ESwab ${ }^{\mathrm{TM}}$ collection and transport system (ESwab; Becton, Dickinson and Company, Sparks, MD). All swabs were transported at $2-4{ }^{\circ} \mathrm{C}$. All swabs and transport systems were used according to the manufacturers' instructions. Affirm, MAX VP, and the Hologic Aptima ${ }^{\circledR}$ Trichomonas vaginalis (Aptima TV; Hologic Inc., San Diego, CA) assay were used according to the manufacturers' instructions.

For Candida culture, vaginal ESwab specimens were collected and transported to the laboratory according to the manufacturer's instructions and processed within $24-48 \mathrm{~h}$ of collection. BD BBLTM CHROMagar ${ }^{\mathrm{TM}}$ Candida medium (CHROMagar) and BD BBLTM Sabouraud Dextrose Agar, Emmons (SDA-Emmons) plates were inoculated with $100 \mu \mathrm{L}$ of the liquid Amies medium. The CHROMagar plate was incubated at $33-37^{\circ} \mathrm{C}$ and read after $36-48 \mathrm{~h}$. The SDAEmmons plate was incubated at $25-30^{\circ} \mathrm{C}$ and read after $36-$ $48 \mathrm{~h}$ and after $64-80 \mathrm{~h}$ of incubation. Candida isolates were identified using Bruker MALDI Biotyper®.

For Gram stain and Nugent score, a $50-\mu \mathrm{L}$ aliquot of the vaginal ESwab specimen used for Candida culture was used to prepare a smear for Gram staining. Stained slides were evaluated by a single technologist for bacterial vaginosis using the Nugent score criteria (negative $0-3$, indeterminate 4-6, positive $\geq 7$ ) [17].

Chart review of patient clinical data was performed for all patients in the study to determine clinical signs and symptoms of vaginal infection: symptoms of vaginitis, urinary symptoms, abdominal symptoms, prior antibiotic or antifungal treatment, date and duration of therapy, other sexually transmitted infections, and clinician's evaluation and diagnosis.

\section{Data analysis}

Samples with discordant test results between MAX VP and Affirm were adjudicated by the following methods: Candida was confirmed using Candida culture, BV was confirmed using the Nugent score criteria, and T. vaginalis was confirmed with Aptima Trichomonas vaginalis assay. A composite reference standard was used to assess the performance of Affirm and MAX VP. A true positive was defined as a positive result for both MAX VP and Affirm or a positive test result from adjudication of discordant results by the Nugent score, Candida culture, or Aptima TV assay. Sensitivity, specificity, positive predictive value (PPV), negative predictive value (NPV), and overall percent agreement (OPA) were calculated for each target following comparison to the composite reference (which includes adjudication of discordant results). Fisher's exact test was used to calculate the statistical significance of sensitivity and specificity. 
Table 1 Demographics for 200 compliant patients included in the study
Patient demographics

\begin{tabular}{|c|c|c|}
\hline \multirow[t]{2}{*}{ Age } & Mean & Median (range) \\
\hline & 30.5 & $29(18-77)$ \\
\hline Race/ethnicity & Patients & $\%$ \\
\hline White & 71 & 36 \\
\hline Non-White/Hispanic & 78 & 39 \\
\hline White/Hispanic & 17 & 9 \\
\hline Native American & 14 & 7 \\
\hline Black/African American & 8 & 4 \\
\hline Asian & 6 & 3 \\
\hline Other/did not answer & 6 & 3 \\
\hline Clinical symptoms & $N$ & $\%$ \\
\hline Discharge & 110 & 55 \\
\hline Itching & 99 & 50 \\
\hline Burning & 56 & 28 \\
\hline Odor & 86 & 43 \\
\hline Dysuria & 27 & 14 \\
\hline Pyuria & 9 & 5 \\
\hline Abdominal pain & 15 & 8 \\
\hline Abdominal cramping & 24 & 12 \\
\hline Other & 25 & 13 \\
\hline \multicolumn{3}{|l|}{ Symptoms } \\
\hline \multirow[t]{8}{*}{ Symptoms per patient } & Number of symptoms & $N(\%)$ \\
\hline & Not documented & $11(5.5)$ \\
\hline & 1 & $49(24.5)$ \\
\hline & 2 & $65(32.5)$ \\
\hline & 3 & $42(21.0)$ \\
\hline & 4 & $19(9.5)$ \\
\hline & 5 & $13(6.5)$ \\
\hline & 6 & $1(0.5)$ \\
\hline Average symptoms/patient & & 2.1 \\
\hline Average duration of symptoms (days) & & 19.7 \\
\hline \multicolumn{3}{|l|}{ Other causes of symptoms } \\
\hline & Patients & $\%$ \\
\hline Chlamydia & 3 & 1.5 \\
\hline Gonorrhea & 1 & 0.5 \\
\hline Genital HSV & 1 & 0.5 \\
\hline Urinary tract infection & 13 & 6.5 \\
\hline \multicolumn{3}{|l|}{ Treatment based on standard-of-care result } \\
\hline & Positive test result & Treated, $N(\%)$ \\
\hline BV & 95 & 87 (91.6) \\
\hline VVC & 43 & $32(74.4)$ \\
\hline TV & 8 & $7(87.5)$ \\
\hline
\end{tabular}

$H S V$ herpes simplex virus, $B V$ bacterial vaginosis, $V V C$ vulvovaginal candidiasis, $T V$ Trichomonas vaginalis

\section{Results}

\section{Subject population}

Among the 200 compliant subjects, the age range was 18 77 years with a mean age of 30.5 years. Patients often presented with multiple symptoms, the most frequent of which included discharge (55\%), itching (50\%), and odor (43\%). The average duration of symptoms was 19.7 days; 13 patients documented $>6$ months of symptom duration (Table 1). When evaluating both Affirm and MAX VP, 20\% (40/200 subjects) were negative for all targets tested. BV was the most 
prevalent condition detected $(41.6 \% ; 79 / 190)$, followed by Candida spp. (32.1\%; 62/193) and T. vaginalis $(4.2 \%$; 8/192) (Table 2).

\section{Bacterial vaginosis}

Patients positive for BV most frequently presented with symptoms of odor $(55.7 \%$; 44/79), discharge $(55.7 \%$; 44/79), and itching $(46.8 \%$; 437/79). When the duration of symptoms could be documented, BV-positive patients reported an average of 22.2 days of symptoms prior to seeking care; six patients reported $>6$ months of symptom duration. MAX VP performed significantly better than Affirm with a specificity of $96.1 \%$ and only $5.1 \%$ (4/79) false-positive results (Table 2). The specificity of Affirm-GV was $81.6 \%$. There were 29 Affirm-GV positive/MAX VP-BV negative samples. Of these patients, $96.6 \%$ (28/29) were treated for BV based on the positive Affirm-GV result (Table 3). Following discordant resolution of these samples by Nugent score criteria, only $10.3 \%(3 / 29)$ were true positive, $65.5 \%(19 / 29)$ were true negative, and $24.1 \%(7 / 29)$ were indeterminate (Table 3$)$. All 19 patients with false-positive Affirm results were treated for BV based on these standard-of-care results and clinical presentation. Eight patients were MAX VP-BV positive/ Affirm-GV negative; $50 \%$ (4/8) were true negative, $37.5 \%$ $(3 / 8)$ were true positive, and $12.5 \%(1 / 8)$ were indeterminate by Nugent score criteria (Table 3 ). Of the MAX VP-BV positive patients with negative Affirm result, one patient was diagnosed and clinically treated for presumed BV despite the negative standard-of-care results. Two additional patients had a history of recurrent BV.

\section{Candida species}

Patients who were positive for Candida spp. most commonly presented with itching $(71.0 \%$; 44/66), discharge $(62.9 \%$; 39/ 66), and burning (38.7\%; 24/66). Patients with Candida spp. had symptoms for an average of 23.7 days prior to presenting to care, with an additional 4 having symptoms for greater than 6 months. The performance characteristics of Affirm and MAX VP for the detection of Candida spp. were evaluated. The Affirm-Candida result does not differentiate $C$. albicans, C. glabrata, C. kefyr, C. krusei, C. parapsilosis, and C. tropicalis while the MAX VP assay (MAX VP-Candida) differentiates Candida group, C. glabrata, and C. krusei. For the initial analysis, detection of Candida species for MAX VP-Candida was not differentiated by species and the results are presented in Table 2. Affirm-Candida had a sensitivity, specificity, NPV, and PPV of $69.4 \%, 87.3 \%, 100 \%$, and $100 \%$, respectively, compared to $98.4 \%, 99.2 \%, 95.4 \%$, and 91.4\% for MAX VP-Candida (Table 2). When Candida species were differentiated using MAX VP results, there were 63 Candida group, 9 C. glabrata, and 0 C. krusei detected (Table 4). For MAX VP-Candida, 88.9\% (56/63) of the Candida group (55 C. albicans and 1 C. dubliniensis) were confirmed in culture for an overall percent agreement with culture of $95.9 \%$ (98.3\% positive agreement and $94 \%$ negative agreement) (Table 4).

For C. glabrata, $88.9 \%(8 / 9)$ of the samples positive by MAX VP were confirmed by culture for an overall agreement of $99.5 \%$ (100\% positive agreement, $99.5 \%$ negative agreement) with culture (Table 4). Of the patients who were positive for Candida using the standard-of-care Affirm test, eight had true-positive C. glabrata as determined by culture and MAX VP and six were treated with fluconazole based on the Affirm result.

\section{Trichomonas}

The performance characteristics of Affirm and MAX VP for the detection of Trichomonas vaginalis were evaluated. There was $100 \%$ concordance between Affirm-TV and MAX VPTV. Seven of the eight patients who were TV-positive by MAX VP were also positive for BV.

Table 2 Overall performance of BD MAX vaginal panel and BD Affirm VPIII assays

\begin{tabular}{|c|c|c|c|c|c|c|c|c|c|c|c|}
\hline & \multirow[t]{2}{*}{ Prevalence } & \multirow[t]{2}{*}{$\mathrm{TP}$} & \multirow[t]{2}{*}{$\mathrm{FP}$} & \multirow[t]{2}{*}{$\mathrm{TN}$} & \multirow[t]{2}{*}{$\mathrm{FN}$} & \multicolumn{2}{|c|}{ Sensitivity } & \multicolumn{2}{|c|}{ Specificity } & \multirow{2}{*}{$\begin{array}{l}\text { PPV } \\
\%\end{array}$} & \multirow{2}{*}{$\begin{array}{l}\text { NPV } \\
\%\end{array}$} \\
\hline & & & & & & $\%$ & $95 \% \mathrm{CI}$ & $\%$ & $95 \% \mathrm{CI}$ & & \\
\hline MAX VP-BV & $41.6 \%{ }^{\dagger}$ & 76 & 4 & 99 & 3 & 96.2 & $89.3-99.2$ & 96.1 & $89.8-98.7$ & 95.0 & 97.1 \\
\hline Affirm-GV & & 76 & 19 & 84 & 3 & 96.2 & $89.5-99.2$ & 81.6 & $72.7-88.5$ & 80.0 & 96.6 \\
\hline MAX VP-Candida & $32.1 \%$ & 61 & 6 & 125 & 1 & 98.4 & $91.3-99.6$ & 95.4 & $90.3-98.3$ & 91.4 & 99.2 \\
\hline Affirm-Candida & & 43 & 0 & 131 & 19 & 69.4 & $56.4-80.4$ & 100.0 & $97.2-100$ & 100.0 & 87.3 \\
\hline
\end{tabular}

Total percent positive agreement $97.3 \%$, percent negative agreement $97.8 \%$, percent overall agreement $97.7 \%$; Affirm percent positive agreement $85.9 \%$, percent negative agreement $95.5 \%$, percent overall agreement $94.1 \%$. $P<0.001$ for the performance of MAX VP compared to the Affirm

$B V$ bacterial vaginosis, $M A X V P$ BD MAX Vaginal Panel, $T P$ true positive, $F P$ false positive, $T N$ true negative, $F N$ false negative, $P P V$ positive predictive value, $N P V$ negative predictive value

$\dagger 8$ specimens were indeterminate by Nugent score criteria 
Table 3 Discordant analysis for the detection of bacterial vaginosis

\begin{tabular}{llllc}
\hline & Nugent positive & Nugent negative & Nugent indeterminate & Treated for BV, $N(\%)$ \\
\hline $\begin{array}{l}\text { Affirm positive/ } \\
\text { MAX VP negative }\end{array}$ & 3 & 19 & 7 & $28 / 29(96.5)$ \\
$\begin{array}{l}\text { Affirm negative/ } \\
\text { MAX VP positive }\end{array}$ & 3 & 4 & 1 & $1 / 8(12.5)$ \\
\hline
\end{tabular}

Discordant analysis for the detection of organisms associated with bacterial vaginosis or normal flora. Nugent scoring criteria were used to adjudicate discordant results. Nugent negative $0-3$, indeterminate $4-6$, positive $\geq 7$

\section{Discussion}

The results of this study demonstrate that MAX VP provides better specificity for the detection of BV compared to Affirm with no difference in sensitivity, whereas it provides a superior sensitivity, with specificity that is at least as good, when compared to Affirm for the detection of Candida spp.; an equivalent performance for TV in symptomatic patients was observed for MAX VP compared to Affirm.

The prevalence of BV in this study was $41.6 \%$; other studies report a BV prevalence of about $20-64 \%$ in symptomatic women $[15,18]$. Affirm had a specificity $(81.6 \%)$ for BV that is consistent with previous reports $[15,19]$. MAX VP was associated with a significantly better specificity of $96.1 \%$ compared to Affirm. The difference in specificity for these two assays is likely due to the detection of $G$. vaginalis alone in Affirm compared to the algorithmic detection of a combination of markers in the MAX VP assay. The inclusion of Lactobacillus spp. as an indicator of normal flora and the presence of G. vaginalis, A. vaginae, Megasphaera-1, and BVAB-2 as indicators of BV in MAX VP increase the specificity for the detection of BV. The sensitivity for both assays was identical (96.2\%), with each having 3 false-negative results.

For VVC, $29.0 \%$ of infections were caused by Candida group and $4.1 \%$ of infections were caused by C. glabrata; there were no $C$. krusei detected in the study, and the overall Candida spp. prevalence was $32.1 \%$. Overall, MAX VP significantly outperformed Affirm with a sensitivity of $98.4 \%$ compared to $69.4 \%$. An additional 18 true-positive samples were detected using MAX VP. Although Affirm does not differentiate Candida spp., it detects C. albicans, C. glabrata, C. kefyr, C. krusei, C. parapsilosis, and C. tropicalis. Therefore, only the one sample containing C. dubliniensis would not have been detected in Affirm based on the absence of target coverage.

MAX VP differentiates C. glabrata and C. krusei, which are important causes of VVC, but clinically indistinguishable from C. albicans. Speciation of Candida for VVC may be important as $50 \%$ of C. glabrata isolates from VVC have decreased sensitivity to fluconazole and C. krusei is intrinsically resistant to the antifungal agent. Although optimal therapy for non-albicans VVC has not been identified, longer duration with a non-fluconazole azole regimen or use of a vaginal boric acid capsule is recommended [1]. C. glabrata and other non-albicans Candida species have also been recovered in $10-20 \%$ of women with recurrent VVC (RVVC), which is defined as four or more episodes of symptomatic VVC within 1 year [20]. The data for treatment of nonalbicans VVC is likely limited due to the lack of testing and differentiation of non-albicans isolates. Increased recognition of the prevalence of non-albicans VVC and their role in RVVC may provide additional data to optimize treatment choices.

This study has some limitations. The patient sample size was relatively small which likely impacted the detection of TV and the assessment of differences between Affirm-TV and MAX VP-TV. Although TV infections were limited in this study population, it is important to detect this pathogen in the differential diagnosis and treatment of vaginitis since patient symptoms often overlap. Moreover, these findings are consistent with other studies showing that co-infections with
Table 4 Performance of BD MAX Vaginal Panel for detection of Candida spp. after discordant analysis

\begin{tabular}{lrrrrrrrl}
\hline & $N$ & TP & FP & TN & FN & PPA & NPA & Overall agreement \\
\hline Candida group & 63 & $56^{\dagger}$ & $7^{\dagger \dagger}$ & 129 & 1 & $98.3 \%$ & $94.9 \%$ & $95.9 \%$ \\
Candida glabrata & 9 & 8 & $1^{*}$ & 184 & 0 & $100.0 \%$ & $99.5 \%$ & $99.5 \%$ \\
\hline
\end{tabular}

Performance of MAX VP for detection and differentiation of Candida. Candida group includes C. albicans, C. tropicalis, C. parapsilosis, C. dubliniensis, C. glabrata, and C. krusei

$T P$ true positive, $F P$ false positive, $T N$ true negative, $F N$ false negative, $P P A$ positive percent agreement, $N P A$ negative percent agreement

${ }^{\dagger}$ Organisms recovered: 55 C. albicans, 1 C. dubliniensis

${ }^{\dagger}$ Organisms recovered: 1 C. glabrata, 6 no Candida isolated

*Organisms recovered: 1 C. albicans 
TV and BV can occur [21], which underscore the need for accurate diagnosis. A larger study may be important to understand the prevalence of TV and the extent or impact of coinfections involving TV, BV, or Candida on treatment or patient management in this study population. While $C$. glabrata was identified in this study, antifungal susceptibility testing was not performed to determine if the efficacy of empiric fluconazole treatment would be impacted by the detection of this organism. Overall, when comparing the two commercially available assays for the most common causes of vaginitis, MAX VP had improved performance and diagnostic accuracy for the diagnosis of vaginitis.

Acknowledgments The authors would like to thank the Obstetrics and Gynecology Department of the University of New Mexico and the Infectious Disease Department at TriCore Reference Laboratory.

Funding information The study was supported by BD Life Sciences Diagnostic Systems (Sparks, MD).

\section{Compliance with ethical standards}

Conflict of interest The authors declare that they have no conflicts of interest.

Ethical approval All procedures performed in studies involving human participants were in accordance with the ethical standards of the Institutional Review Board of the University of New Mexico and with the 1964 Helsinki declaration and its later amendments or comparable ethical standards.

Open Access This article is distributed under the terms of the Creative Commons Attribution 4.0 International License (http:// creativecommons.org/licenses/by/4.0/), which permits unrestricted use, distribution, and reproduction in any medium, provided you give appropriate credit to the original author(s) and the source, provide a link to the Creative Commons license, and indicate if changes were made.

\section{References}

1. Workowski KA, Bolan GA (2015) Sexually transmitted diseases treatment guidelines, 2015. MMWR Recomm Rep 64(Rr-03):1137

2. Kent HL (1991) Epidemiology of vaginitis. Am J Obstet Gynecol 165(4 Pt 2):1168-1176

3. Achkar JM, Fries BC (2010) Candida infections of the genitourinary tract. Clin Microbiol Rev 23(2):253-273

4. Kissinger P (2015) Trichomonas vaginalis: a review of epidemiologic, clinical and treatment issues. BMC Infect Dis 15:307-307

5. Ravel J, Gajer P, Abdo Z, Schneider GM, Koenig SS, McCulle SL, Karlebach S, Gorle R, Russell J, Tacket CO, Brotman RM, Davis CC, Ault K, Peralta L, Forney LJ (2011) Vaginal microbiome of reproductive-age women. Proc Natl Acad Sci U S A 108 Suppl 1: $4680-4687$
6. Ravel J, Brotman RM, Gajer P, Ma B, Nandy M, Fadrosh DW, Sakamoto J, Koenig SS, Fu L, Zhou X, Hickey RJ, Schwebke JR, Forney LJ (2013) Daily temporal dynamics of vaginal microbiota before, during and after episodes of bacterial vaginosis. Microbiome 1(1):29

7. Willett LL, Centor RM (2005) Evaluating vaginitis. The importance of patient factors. J Gen Intern Med 20(9):871-871

8. Schwebke JR, Gaydos CA, Nyirjesy P, Paradis S, Kodsi S, Cooper CK (2018) Diagnostic performance of a molecular test versus clinician assessment of vaginitis. J Clin Microbiol 56(6):e00252e00218

9. Carr PL, Felsenstein D, Friedman RH (1998) Evaluation and management of vaginitis. J Gen Intern Med 13(5):335-346

10. Kumar N, Behera B, Sagiri SS, Pal K, Ray SS, Roy S (2011) Bacterial vaginosis: etiology and modalities of treatment-a brief note. J Pharm Bioallied Sci 3(4):496-503

11. Leitich H, Kiss H (2007) Asymptomatic bacterial vaginosis and intermediate flora as risk factors for adverse pregnancy outcome. Best Pract Res Clin Obstet Gynaecol 21(3):375-390

12. Atashili J, Poole C, Ndumbe PM, Adimora AA, Smith JS (2008) Bacterial vaginosis and HIV acquisition: a meta-analysis of published studies. AIDS (London, England) 22(12):1493-1501

13. Balkus JE, Richardson BA, Rabe LK, Taha TE, Mgodi N, Kasaro MP, Ramjee G, Hoffman IF, Abdool Karim SS (2014) Bacterial vaginosis and the risk of trichomonas vaginalis acquisition among HIV-1-negative women. Sex Transm Dis 41(2):123-128

14. Fastring DR, Amedee A, Gatski M, Clark RA, Mena LA, Levison J, Schmidt N, Rice J, Gustat J, Kissinger P (2014) Co-occurrence of Trichomonas vaginalis and bacterial vaginosis and vaginal shedding of HIV-1 RNA. Sex Transm Dis 41(3):173-179

15. Cartwright CP, Lembke BD, Ramachandran K, Body BA, Nye MB, Rivers CA, Schwebke JR (2013) Comparison of nucleic acid amplification assays with BD affirm VPIII for diagnosis of vaginitis in symptomatic women. J Clin Microbiol 51(11):3694-3699

16. Aguirre-Quinonero A, Castillo-Sedano IS, Calvo-Muro F, CanutBlasco A (2019) Accuracy of the BD MAX vaginal panel in the diagnosis of infectious vaginitis. Eur J Clin Microbiol Infect Dis 38(5):877-882

17. Nugent RP, Krohn MA, Hillier SL (1991) Reliability of diagnosing bacterial vaginosis is improved by a standardized method of gram stain interpretation. J Clin Microbiol 29(2):297-301

18. Witt A, Petricevic L, Kaufmann U, Gregor H, Kiss H (2002) DNA hybridization test: rapid diagnostic tool for excluding bacterial vaginosis in pregnant women with symptoms suggestive of infection. J Clin Microbiol 40(8):3057-3059

19. Brown HL, Fuller DD, Jasper LT, Davis TE, Wright JD (2004) Clinical evaluation of affirm VPIII in the detection and identification of trichomonas vaginalis, Gardnerella vaginalis, and Candida species in vaginitis/vaginosis. Infect Dis Obstet Gynecol 12(1):1721

20. Sobel JD (2016) Recurrent vulvovaginal candidiasis. Am J Obstet Gynecol 214(1):15-21

21. Gaydos CA, Beqaj S, Schwebke JR, Lebed J, Smith B, Davis TE, Fife KH, Nyirjesy P, Spurrell T, Furgerson D, Coleman J, Paradis S, Cooper CK (2017) Clinical validation of a test for the diagnosis of vaginitis. Obstet Gynecol 130(1):181-189

Publisher's note Springer Nature remains neutral with regard to jurisdictional claims in published maps and institutional affiliations. 